Stud. Univ. Babeş-Bolyai Math. 64(2019), No. 3, 357-365

DOI: http://dx.doi.org/10.24193/subbmath.2019.3.07

\title{
Approximation with Riemann-Liouville fractional derivatives
}

\author{
George A. Anastassiou
}

\begin{abstract}
In this article we study quantitatively with rates the pointwise convergence of a sequence of positive sublinear operators to the unit operator over continuous functions. This takes place under low order smothness, less than one, of the approximated function and it is expressed via the left and right RiemannLiouville fractional derivatives of it. The derived related inequalities in their right hand sides contain the moduli of continuity of these fractional derivatives and they are of Shisha-Mond type. We give applications to Bernstein Max-product operators and to positive sublinear comonotonic operators connecting them to Choquet integral.
\end{abstract}

Mathematics Subject Classification (2010): 26A33, 41A17, 41A25, 41A36, 41A80.

Keywords: Riemann-Liouville fractional derivative, positive sublinear operators, modulus of continuity, comonotonic operator, Choquet integral.

\section{Introduction}

In this paper among others we are motivated by the following results:

First by P.P. Korovkin [9], (1960), p. 14: Let $[a, b]$ be a closed interval in $\mathbb{R}$ and $\left(L_{n}\right)_{n \in \mathbb{N}}$ be a sequence of positive linear operators mapping $C([a, b])$ into itself. Suppose that $\left(L_{n} f\right)$ converges uniformly to $f$ for the three test functions $f=1, x, x^{2}$. Then $\left(L_{n} f\right)$ converges uniformly to $f$ on $[a, b]$ for all functions $f \in C([a, b])$.

Let $f \in C([a, b])$ and $0 \leq h \leq b-a$. The first modulus of continuity of $f$ at $h$ is given by

$$
\omega_{1}(f, h)=\sup _{\substack{x, y \in[a, b] \\|x-y| \leq h}}|f(x)-f(y)| .
$$

If $h>b-a$, then we define $\omega_{1}(f, h)=\omega_{1}(f, b-a)$.

Another motivation is the following: 
By Shisha and Mond [12], (1968): Let $[a, b] \subset \mathbb{R}$ a closed interval. Let $\left\{L_{n}\right\}_{n \in \mathbb{N}}$ be a sequence of positive linear operators acting on $C([a, b])$ into itself. For $n=1, \ldots$, suppose $L_{n}(1)$ is bounded. Let $f \in C([a, b])$. Then for $n=1,2, \ldots$, we have

$$
\left\|L_{n} f-f\right\|_{\infty} \leq\|f\|_{\infty}\left\|L_{n} 1-1\right\|_{\infty}+\left\|L_{n} 1+1\right\|_{\infty} \omega_{1}\left(f, \mu_{n}\right),
$$

where

$$
\mu_{n}=\left\|L_{n}\left((t-x)^{2}\right)(x)\right\|_{\infty}^{\frac{1}{2}}
$$

and $\|\cdot\|_{\infty}$ stands for the sup-norm over $[a, b]$.

One can easily see, for $n=1,2, \ldots$

$$
\mu_{n}^{2} \leq\left\|L_{n}\left(t^{2} ; x\right)-x^{2}\right\|_{\infty}+2 c\left\|L_{n}(t ; x)-x\right\|_{\infty}+c^{2}\left\|L_{n}(1 ; x)-1\right\|_{\infty},
$$

where $c=\max (|a|,|b|)$.

Thus, given the Korovkin assumptions, as $n \rightarrow \infty$, we get $\mu_{n} \rightarrow 0$, and $\left\|L_{n} f-f\right\|_{\infty} \rightarrow 0$ for any $f \in C([a, b])$. That is one derives the Korovkin conclusion in a quantitative way and with rates of convergence.

We continue this type as research here for positive sublinear operators over continuous functions with existing left and right Riemann-Liouville fractional derivatives of order less than one. We give applications.

Other motivations come from author's monographs [2], [3] and [4].

\section{Main results}

We mention

Definition 2.1. ([10, pp. 68, 89]) Let $x, x^{\prime} \in[a, b], f \in C([a, b])$. The Riemann-Liouville (R-L) fractional derivative of a function $f$ of order $q(0<q<1)$ is defined as

$$
\begin{gathered}
D_{x}^{q} f\left(x^{\prime}\right)=\left\{\begin{array}{cc}
D_{x+}^{q} f\left(x^{\prime}\right), & x^{\prime}>x, \\
D_{x-}^{q} f\left(x^{\prime}\right), & x^{\prime}<x
\end{array}\right\} \\
=\frac{1}{\Gamma(1-q)}\left\{\begin{array}{c}
\frac{d}{d x^{\prime}} \int_{x}^{x^{\prime}}\left(x^{\prime}-t\right)^{-q} f(t) d t, \quad x^{\prime}>x, \\
-\frac{d}{d x^{\prime}} \int_{x^{\prime}}^{x}\left(t-x^{\prime}\right)^{-q} f(t) d t, \quad x^{\prime}<x,
\end{array}\right.
\end{gathered}
$$

the left and right $\mathrm{R}-\mathrm{L}$ fractional derivatives, respectively, where $\Gamma$ is the gamma function.

We need

Lemma 2.2. ([1], [10], pp. 71, 75) Let $x, x^{\prime} \in[a, b], f \in C([a, b]), 0<q<1$. Assume that $D_{x+}^{q}(f(\cdot)-f(x)) \in C([x, b]), D_{x-}^{q}(f(\cdot)-f(x)) \in C([a, x])$, where $x$ is fixed. Then

all $x<x^{\prime} \leq b$, and

$$
f\left(x^{\prime}\right)-f(x)=\frac{1}{\Gamma(q)} \int_{x}^{x^{\prime}}\left(x^{\prime}-z\right)^{q-1} D_{x+}^{q}(f(z)-f(x)) d z
$$

$$
f\left(x^{\prime}\right)-f(x)=\frac{1}{\Gamma(q)} \int_{x^{\prime}}^{x}\left(z-x^{\prime}\right)^{q-1} D_{x-}^{q}(f(z)-f(x)) d z,
$$

all $a \leq x^{\prime}<x$. 
We accept $0 \cdot \infty=0$ and we notice that $D_{x+}^{q} 0=D_{x-}^{q} 0=0$.

We need

Definition 2.3. Let $f \in C([a, b])$. The first modulus of continuity is given by

$$
\omega_{1}(f, \delta):=\sup _{\substack{x, y \in[a, b] \\|x-y| \leq \delta}}|f(x)-f(y)|, \quad \delta>0 .
$$

We need

Definition 2.4. Denote by $D_{x}^{q}(f(\cdot)-f(x))$ any of $D_{x \pm}^{q}(f(\cdot)-f(x))$, and $\delta>0$.

We set

$$
\begin{gathered}
\omega_{1}\left(D_{x}^{q}(f(\cdot)-f(x)), \delta\right) \\
:=\max \left\{\omega_{1}\left(D_{x+}^{q}(f(\cdot)-f(x)), \delta\right)_{[x, b]}, \omega_{1}\left(D_{x-}^{q}(f(\cdot)-f(x)), \delta\right)_{[a, x]}\right\} .
\end{gathered}
$$

We give

Theorem 2.5. Here $f \in C([a, b]), 0<q<1, \delta>0 ; x, x^{\prime} \in[a, b]$. Assume that $D_{x+}^{q}(f(\cdot)-f(x)) \in C([x, b])$, and $D_{x-}^{q}(f(\cdot)-f(x)) \in C([a, x])$, where $x$ is fixed. Then

$$
\left|f\left(x^{\prime}\right)-f(x)\right| \leq \frac{\omega_{1}\left(D_{x}^{q}(f(\cdot)-f(x)), \delta\right)}{\Gamma(q+1)}\left[\left|x^{\prime}-x\right|^{q}+\frac{\left|x^{\prime}-x\right|^{q+1}}{(q+1) \delta}\right],
$$

$\forall x^{\prime} \in[a, b]$.

Proof. Obviously $D_{x+}^{q}(f(x)-f(x))=0$. We estimate:

i) Case of $x<x^{\prime} \leq b$ :

$$
\begin{aligned}
& \left|f\left(x^{\prime}\right)-f(x)\right| \leq \frac{1}{\Gamma(q)} \int_{x}^{x^{\prime}}\left(x^{\prime}-z\right)^{q-1}\left|D_{x+}^{q}(f(z)-f(x))\right| d z \\
= & \frac{1}{\Gamma(q)} \int_{x}^{x^{\prime}}\left(x^{\prime}-z\right)^{q-1}\left|D_{x+}^{q}(f(z)-f(x))-D_{x+}^{q}(f(x)-f(x))\right| d z \\
& \quad \leq \frac{\left(\delta_{1}>0\right)}{\Gamma(q)} \int_{x}^{x^{\prime}}\left(x^{\prime}-z\right)^{q-1} \omega_{1}\left(D_{x+}^{q}(f(\cdot)-f(x)), \frac{\delta_{1}(z-x)}{\delta_{1}}\right)_{[x, b]} d z \\
\leq & \frac{1}{\Gamma(q)} \omega_{1}\left(D_{x+}^{q}(f(\cdot)-f(x)), \delta_{1}\right)_{[x, b]}\left(\int_{x}^{x^{\prime}}\left(x^{\prime}-z\right)^{q-1}\left(1+\frac{z-x}{\delta_{1}}\right) d z\right) \\
= & \frac{\omega_{1}\left(D_{x+}^{q}(f(\cdot)-f(x)), \delta_{1}\right)_{[x, b]}\left[\frac{\left(x^{\prime}-x\right)^{q}}{q}+\frac{1}{\delta_{1}} \int_{x}^{x^{\prime}}\left(D^{\prime}-z\right)^{q-1}(z-x)^{2-1} d z\right]}{\left.\Gamma(q)-f(x)), \delta_{1}\right)_{[x, b]}\left[\frac{\left(x^{\prime}-x\right)^{q}}{q}+\frac{1}{\delta_{1}} \frac{\Gamma(q) \Gamma(2)}{\Gamma(q+2)}\left(x^{\prime}-x\right)^{q+1}\right]} \\
= & \left.\frac{\omega_{1}\left(D_{x+}^{q}(f(\cdot)-f(x)), \delta_{1}\right)_{[x, b]}\left[\frac{\left(x^{\prime}-x\right)^{q}}{q}+\frac{1}{\delta_{1}} \frac{\Gamma(q)}{\Gamma(q+2)}\left(x^{\prime}-x\right)^{q+1}\right]}{\Gamma(q)}\right]
\end{aligned}
$$




$$
\begin{aligned}
& =\frac{\omega_{1}\left(D_{x+}^{q}(f(\cdot)-f(x)), \delta_{1}\right)_{[x, b]}}{\Gamma(q)}\left[\frac{\left(x^{\prime}-x\right)^{q}}{q}+\frac{1}{\delta_{1}} \frac{\left(x^{\prime}-x\right)^{q+1}}{q(q+1)}\right] \\
& =\frac{\omega_{1}\left(D_{x+}^{q}(f(\cdot)-f(x)), \delta_{1}\right)_{[x, b]}}{\Gamma(q+1)}\left[\left(x^{\prime}-x\right)^{q}+\frac{1}{\delta_{1}} \frac{\left(x^{\prime}-x\right)^{q+1}}{(q+1)}\right] .
\end{aligned}
$$

When $x<x^{\prime} \leq b$, we have proved that

$$
\begin{gathered}
\left|f\left(x^{\prime}\right)-f(x)\right| \leq \\
\frac{\omega_{1}\left(D_{x+}^{q}(f(\cdot)-f(x)), \delta_{1}\right)_{[x, b]}}{\Gamma(q+1)}\left[\left(x^{\prime}-x\right)^{q}+\frac{\left(x^{\prime}-x\right)^{q+1}}{(q+1) \delta_{1}}\right],
\end{gathered}
$$

where $0<q<1, \delta_{1}>0$.

ii) Case of $a \leq x^{\prime}<x$ (here $\left.D_{x-}^{q}(f(x)-f(x))=0\right)$ :

$$
\begin{aligned}
& \left|f\left(x^{\prime}\right)-f(x)\right| \leq \frac{1}{\Gamma(q)} \int_{x^{\prime}}^{x}\left(z-x^{\prime}\right)^{q-1}\left|D_{x-}^{q}(f(z)-f(x))\right| d z \\
& =\frac{1}{\Gamma(q)} \int_{x^{\prime}}^{x}\left(z-x^{\prime}\right)^{q-1}\left|D_{x-}^{q}(f(z)-f(x))-D_{x-}^{q}(f(x)-f(x))\right| d z \\
& \stackrel{\left(\delta_{2}>0\right)}{\leq} \frac{1}{\Gamma(q)} \int_{x^{\prime}}^{x}\left(z-x^{\prime}\right)^{q-1} \omega_{1}\left(D_{x-}^{q}(f(\cdot)-f(x)), \frac{\delta_{2}(x-z)}{\delta_{2}}\right)_{[a, x]} d z \\
& \leq \frac{\omega_{1}\left(D_{x-}^{q}(f(\cdot)-f(x)), \delta_{2}\right)_{[a, x]}}{\Gamma(q)}\left(\int_{x^{\prime}}^{x}\left(z-x^{\prime}\right)^{q-1}\left(1+\frac{x-z}{\delta_{2}}\right) d z\right) \\
& =\frac{\omega_{1}\left(D_{x-}^{q}(f(\cdot)-f(x)), \delta_{2}\right)_{[a, x]}}{\Gamma(q)}\left[\frac{\left(x-x^{\prime}\right)^{q}}{q}+\frac{1}{\delta_{2}} \int_{x^{\prime}}^{x}(x-z)^{2-1}\left(z-x^{\prime}\right)^{q-1} d z\right] \\
& =\frac{\omega_{1}\left(D_{x-}^{q}(f(\cdot)-f(x)), \delta_{2}\right)_{[a, x]}}{\Gamma(q)}\left[\frac{\left(x-x^{\prime}\right)^{q}}{q}+\frac{1}{\delta_{2}} \frac{\Gamma(2) \Gamma(q)}{\Gamma(q+2)}\left(x-x^{\prime}\right)^{q+1}\right] \\
& =\frac{\omega_{1}\left(D_{x-}^{q}(f(\cdot)-f(x)), \delta_{2}\right)_{[a, x]}}{\Gamma(q)}\left[\frac{\left(x-x^{\prime}\right)^{q}}{q}+\frac{\left(x-x^{\prime}\right)^{q+1}}{q(q+1) \delta_{2}}\right] \\
& =\frac{\omega_{1}\left(D_{x-}^{q}(f(\cdot)-f(x)), \delta_{2}\right)_{[a, x]}}{\Gamma(q+1)}\left[\left(x-x^{\prime}\right)^{q}+\frac{\left(x-x^{\prime}\right)^{q+1}}{(q+1) \delta_{2}}\right] \text {. }
\end{aligned}
$$

When $a \leq x^{\prime}<x$, we have proved that

$$
\begin{gathered}
\left|f\left(x^{\prime}\right)-f(x)\right| \leq \\
\frac{\omega_{1}\left(D_{x-}^{q}(f(\cdot)-f(x)), \delta_{2}\right)_{[a, x]}}{\Gamma(q+1)}\left[\left(x-x^{\prime}\right)^{q}+\frac{\left(x-x^{\prime}\right)^{q+1}}{(q+1) \delta_{2}}\right],
\end{gathered}
$$

where $0<q<1, \delta_{2}>0$.

Finally choose: $\delta_{1}=\delta_{2}=: \delta>0$. The theorem is proved.

We need 
Definition 2.6. Here $C_{+}([a, b]):=\left\{f:[a, b] \rightarrow \mathbb{R}_{+}\right.$, continuous functions $\}$. Let $L_{N}: C_{+}([a, b]) \rightarrow C_{+}([a, b])$, operators, $\forall N \in \mathbb{N}$, such that

(i) $L_{N}(\alpha f)=\alpha L_{N}(f), \forall \alpha \geq 0, \forall f \in C_{+}([a, b])$,

(ii) if $f, g \in C_{+}([a, b]): f \leq g$, then $L_{N}(f) \leq L_{N}(g), \forall N \in \mathbb{N}$,

(iii) $L_{N}(f+g) \leq L_{N}(f)+L_{N}(g), \quad \forall f, g \in C_{+}([a, b])$.

We call $\left\{L_{N}\right\}_{N \in \mathbb{N}}$ positive sublinear operators.

We make

Remark 2.7. Let $f, g \in C_{+}([a, b])$, then it holds

$$
\left|L_{N}(f)(x)-L_{N}(g)(x)\right| \leq L_{N}(|f-g|)(x), \forall x \in[a, b] .
$$

Furthermore, we also have

$$
\left|L_{N}(f)(x)-f(x)\right| \leq L_{N}(|f(\cdot)-f(x)|)(x)+|f(x)|\left|L_{N}\left(e_{0}\right)(x)-1\right|,
$$

$\forall x \in[a, b] ; e_{0}(t)=1$.

From now on we assume that $L_{N}(1)=1$. Hence

$$
\left|L_{N}(f)(x)-f(x)\right| \leq L_{N}(|f(\cdot)-f(x)|)(x), \quad \forall x \in[a, b] .
$$

We give

Theorem 2.8. Let $f \in C_{+}([a, b]), 0<q<1, D_{x+}^{q}(f(\cdot)-f(x)) \in C([x, b])$, $D_{x-}^{q}(f(\cdot)-f(x)) \in C([a, x]), x$ is fixed, where $x \in[a, b]$. Then

$$
|f(\cdot)-f(x)| \leq \frac{\omega_{1}\left(D_{x}^{q}(f(\cdot)-f(x)), \delta\right)}{\Gamma(q+1)}\left[|\cdot-x|^{q}+\frac{|\cdot-x|^{q+1}}{(q+1) \delta}\right], \quad \delta>0 .
$$

We present:

Theorem 2.9. Let $f \in C_{+}([a, b]), D_{x+}^{q}(f(\cdot)-f(x)) \in C([x, b]), D_{x-}^{q}(f(\cdot)-f(x)) \in$ $C([a, x])$, where $x \in[a, b]$ is fixed, $0<q<1, \delta>0$. Let $L_{N}: C_{+}([a, b]) \rightarrow C_{+}([a, b])$, be positive sublinear operators, such that $L_{N}(1)=1, \forall N \in \mathbb{N}$. Then

$$
\begin{aligned}
& \qquad L_{N}(f)(x)-f(x) \mid \\
& \leq \frac{\omega_{1}\left(D_{x}^{q}(f(\cdot)-f(x)), \delta\right)}{\Gamma(q+1)}\left[L_{N}\left(|\cdot-x|^{q}\right)(x)+\frac{L_{N}\left(|\cdot-x|^{q+1}\right)(x)}{(q+1) \delta}\right],
\end{aligned}
$$

$\forall N \in \mathbb{N}$.

We need Hölder's inequality for positive sublinear operators:

Lemma 2.10. ([5], p. 6) Let $L: C_{+}([a, b]) \rightarrow C_{+}([a, b])$, be a positive sublinear operator and $f, g \in C_{+}([a, b])$, furthermore let $p, q>1: \frac{1}{p}+\frac{1}{q}=1$. Assume that $L\left((f(\cdot))^{p}\right)\left(s_{*}\right), L\left((g(\cdot))^{q}\right)\left(s_{*}\right)>0$ for some $s_{*} \in[a, b]$. Then

$$
L(f(\cdot) f(\cdot))\left(s_{*}\right) \leq\left(L\left((f(\cdot))^{p}\right)\left(s_{*}\right)\right)^{\frac{1}{p}}\left(L\left((g(\cdot))^{q}\right)\left(s_{*}\right)\right)^{\frac{1}{q}} .
$$

We make 
Remark 2.11. In Theorem 2.9 we assumed $L_{N}(1)=1, \forall N \in \mathbb{N}$. We further assume that $L_{N}\left(|\cdot-x|^{q+1}\right)(x)>0, \forall N \in \mathbb{N}$, for the fixed $x \in[a, b]$.

Then, by (2.19), we obtain

$$
L_{N}\left(|\cdot-x|^{q}\right)(x) \leq\left(L_{N}\left(|\cdot-x|^{q+1}\right)(x)\right)^{\frac{q}{q+1}}, \quad \forall N \in \mathbb{N} .
$$

We give

Theorem 2.12. All as in Theorem 2.9, plus $L_{N}\left(|\cdot-x|^{q+1}\right)(x)>0, \forall N \in \mathbb{N}$, for $a$ fixed $x \in[a, b]$. Then

$$
\begin{gathered}
\left|L_{N}(f)(x)-f(x)\right| \leq \frac{\omega_{1}\left(D_{x}^{q}(f(\cdot)-f(x)), \delta\right)}{\Gamma(q+1)} \\
\cdot\left(L_{N}\left(|\cdot-x|^{q+1}\right)(x)\right)^{\frac{q}{q+1}}\left[1+\frac{\left(L_{N}\left(|\cdot-x|^{q+1}\right)(x)\right)^{\frac{1}{q+1}}}{(q+1) \delta}\right],
\end{gathered}
$$

$\forall N \in \mathbb{N}$.

Next we choose $\delta:=\left(L_{N}\left(|\cdot-x|^{q+1}\right)(x)\right)^{\frac{1}{q+1}}>0$, to obtain:

Theorem 2.13. All as in Theorem 2.9, plus $L_{N}\left(|\cdot-x|^{q+1}\right)(x)>0, \forall N \in \mathbb{N} ; x \in$ $[a, b]$ is fixed. Then

$$
\begin{gathered}
\left|L_{N}(f)(x)-f(x)\right| \leq \frac{(q+2)}{\Gamma(q+2)} \\
\cdot \omega_{1}\left(D_{x}^{q}(f(\cdot)-f(x)),\left(L_{N}\left(|\cdot-x|^{q+1}\right)(x)\right)^{\frac{1}{q+1}}\right)\left(L_{N}\left(|\cdot-x|^{q+1}\right)(x)\right)^{\frac{q}{q+1}},
\end{gathered}
$$

$\forall N \in \mathbb{N}$.

Application 2.14. The max-product Bernstein operators are defined by

$$
B_{N}^{(M)}(f)(x):=\frac{\vee_{k=0}^{N} p_{N . k}(x) f\left(\frac{k}{N}\right)}{\bigvee_{k=0}^{N} p_{N . k}(x)}, \forall N \in \mathbb{N},
$$

where $\vee$ stands for maximum, and $p_{N, k}(x)=\left(\begin{array}{c}N \\ k\end{array}\right) x^{k}(1-x)^{N-k}$, and $f:[0,1] \rightarrow$ $\mathbb{R}_{+}$is a continuous function, see [6], p. 10 .

These are positive sublinear operators mapping $C_{+}([0,1])$ into itself. Notice $B_{N}^{(M)}(1)=1, \forall N \in \mathbb{N}$.

In [5], p. 76, we proved that

$$
B_{N}^{(M)}\left(|\cdot-x|^{1+\beta}\right)(x) \leq \frac{6}{\sqrt{N+1}}, \quad \forall x \in[0,1]
$$

$\forall N \in \mathbb{N}, \forall \beta>0$. 
Furthermore, clearly it holds that

$$
B_{N}^{(M)}\left(|\cdot-x|^{1+\beta}\right)(x)>0, \quad \forall N \in \mathbb{N}, \forall \beta \geq 0,
$$

and any $x \in(0,1)$.

We present

Theorem 2.15. Let $f \in C_{+}([0,1]), \quad D_{x+}^{q}(f(\cdot)-f(x)) \in C([x, 1])$, $D_{x-}^{q}(f(\cdot)-f(x)) \in C([0, x])$, where $x \in(0,1), 0<q<1$. Then

$$
\begin{gathered}
\left|B_{N}^{(M)}(f)(x)-f(x)\right| \\
\leq \frac{(q+2)}{\Gamma(q+2)} \omega_{1}\left(D_{x}^{q}(f(\cdot)-f(x)),\left(\frac{6}{\sqrt{N+1}}\right)^{\frac{1}{q+1}}\right)\left(\frac{6}{\sqrt{N+1}}\right)^{\frac{q}{q+1}},
\end{gathered}
$$

$\forall N \in \mathbb{N}$.

$$
\text { As } N \rightarrow+\infty \text {, we get } B_{N}^{(M)}(f)(x) \rightarrow f(x) \text {. }
$$

Proof. By (2.23), (2.24), (2.25) and Theorem 2.13. task.

One can give many examples like in Theorem 2.15, but we choose to omit it this

Choquet integral has become very important in statistical mechanics, potential theory, non-additive measure theory, and lately in economics. For the definition and properties of Choquet integral read [7], [8], [13].

We denote it by $(C) \int$.

Next we talk about representations of positive sublinear operators by Choquet integrals:

We need

Definition 2.16. Let $\Omega$ be a set, and let $f, g: \Omega \rightarrow \mathbb{R}$ be bounded functions. We say that $f$ and $g$ are comonotonic, if for every $\omega, \omega^{\prime} \in \Omega$,

$$
\left(f(\omega)-f\left(\omega^{\prime}\right)\right)\left(g(\omega)-g\left(\omega^{\prime}\right)\right) \geq 0 .
$$

We also need the famous Schmeidler's Representation Theorem (Schmeidler 1986).

Theorem 2.17. ([11]) Denote with $\mathcal{L}_{\infty}(\mathcal{A})$ the vector space of $\mathcal{A}$-measurable bounded real valued functions on $\Omega$, where $\mathcal{A} \subset 2^{\Omega}$ is a $\sigma$-algebra. Given a real functional $\Gamma: \mathcal{L}_{\infty}(\mathcal{A}) \rightarrow \mathbb{R}$, assume that for $f, g \in \mathcal{L}_{\infty}(\mathcal{A}):$

(i) $\Gamma(c f)=c \Gamma(f), \forall c>0$, and

(ii) $f \leq g$, implies $\Gamma(f) \leq \Gamma(g)$,

(iii) $\Gamma(f+g)=\Gamma(f)+\Gamma(g)$, for any comonotonic $f, g$.

Then $\gamma(A):=\Gamma\left(1_{A}\right), \forall A \in \mathcal{A}$, defines a finite monotone set function on $\mathcal{A}$, and $\Gamma$ is the Choquet integral with respect to $\gamma$, i.e.

$$
\Gamma(f)=(C) \int_{\Omega} f(t) d \gamma(t), \quad \forall f \in \mathcal{L}_{\infty}(\mathcal{A}) .
$$

Above $1_{A}$ denotes the characteristic function on $A$. 
We make

Remark 2.18. Consider here $[a, b] \subset \mathbb{R}, \mathcal{B}=\mathcal{B}([a, b])$ is the Borel $\sigma$-algebra on $[a, b]$, and $\mathcal{L}_{\infty}(\mathcal{B})$ is the vector space of $\mathcal{B}$-measurable bounded real valued functions on $[a, b]$. Let $\left(L_{N}\right)_{N \in \mathbb{N}}$ be a sequence of positive sublinear operators from $\mathcal{L}_{\infty}(\mathcal{B})$ into $C_{+}([a, b])$, and $x \in[a, b]$. That is here $L_{N}$ fulfills the positive homogenuity, monotonicity and subadditivity properties, see Definition 2.6.

Assume $L_{N}(1)=1, \forall N \in \mathbb{N}$. Clearly here $\mathcal{L}_{\infty}(\mathcal{B}) \supset C_{+}([a, b])$. In particular we treat $\left.L_{N}\right|_{C_{+}([a, b])}$, just denoted for simplicity by $L_{N}, \forall N \in \mathbb{N}$.

It is clear that $L_{N}(\cdot)(x): \mathcal{L}_{\infty}(\mathcal{B}) \rightarrow \mathbb{R}$ is a functional, $\forall N \in \mathbb{N}$. It has the properties:

(i)

$$
L_{N}(c f)(x)=c L_{N}(f)(x), \forall c>0, \forall f \in \mathcal{L}_{\infty}(\mathcal{B}),
$$

(ii)

$$
f \leq g \text {, implies } L_{N}(f)(x) \leq L_{N}(g)(x), \text { where } f, g \in \mathcal{L}_{\infty}(\mathcal{B}),
$$

and

(iii)

$$
L_{N}(f+g)(x) \leq L_{N}(f)(x)+L_{N}(g)(x), \quad \forall f, g \in \mathcal{L}_{\infty}(\mathcal{B}) .
$$

For comonotonic $f, g \in \mathcal{L}_{\infty}(\mathcal{B})$, we further assume that

$$
L_{N}(f+g)(x)=L_{N}(f)(x)+L_{N}(g)(x) .
$$

In that case $L_{N}$ is called comonotonic.

By Theorem 2.17 we get that:

$$
\gamma_{N, x}(A):=L_{N}\left(1_{A}\right)(x), \forall A \in \mathcal{B}, \forall N \in \mathbb{N},
$$

defines a finite monotone set function on $\mathcal{B}$, and

$$
L_{N}(f)(x)=(C) \int_{a}^{b} f(t) d \gamma_{N, x}(t),
$$

$\forall f \in \mathcal{L}_{\infty}(\mathcal{B}), \forall N \in \mathbb{N}$.

In particular $(2.34)$ is valid for any $f \in C_{+}([a, b])$. Furthermore $\gamma_{N, x}$ is normalized, that is $\gamma_{N, x}([a, b])=1, \forall N \in \mathbb{N}$.

We give

Theorem 2.19. Let $f \in C_{+}([a, b]), D_{x+}^{q}(f(\cdot)-f(x)) \in C([x, b])$,

$$
D_{x-}^{q}(f(\cdot)-f(x)) \in C([a, x]),
$$

where $x \in[a, b] \subset \mathbb{R}$ is fixed, $0<q<1$. Let $L_{N}: \mathcal{L}_{\infty}(\mathcal{B}([a, b])) \rightarrow C_{+}([a, b])$, be positive sublinear comonotonic operators, such that $L_{N}(1)=1, \forall N \in \mathbb{N}$. Assume that

$$
\text { (C) } \int_{a}^{b}|t-x|^{q+1} d \gamma_{N, x}(t)>0, \forall N \in \mathbb{N}
$$


Then

$$
\begin{aligned}
\left|L_{N}(f)(x)-f(x)\right| & \leq \frac{(q+2)}{\Gamma(q+2)} \omega_{1}\left(D_{x}^{q}(f(\cdot)-f(x)),\left((C) \int_{a}^{b}|t-x|^{q+1} d \gamma_{N, x}(t)\right)^{\frac{1}{q+1}}\right) \\
& \cdot\left((C) \int_{a}^{b}|t-x|^{q+1} d \gamma_{N, x}(t)\right)^{\frac{q}{q+1}}, \forall N \in \mathbb{N} .
\end{aligned}
$$

If

$$
\text { (C) } \int_{a}^{b}|t-x|^{q+1} d \gamma_{N, x}(t) \rightarrow 0
$$

then $L_{N}(f)(x) \rightarrow f(x)$, as $N \rightarrow \infty$.

Proof. By Theorem 2.13.

\section{References}

[1] Adda, F.B., Cresson, J., Fractional differentiation equations and the Schrödinger equation, Applied Math. \& Computation, 161(2005), 323-345.

[2] Anastassiou, G.A., Moments in Probability and Approximation Theory, Pitman Research Notes in Math, Vol. 287, Longman Sci. \& Tech., Harlow, U.K., 1993.

[3] Anastassiou, G.A., Quantitative Approximations, CRC Press, Boca Raton, London, New York, 2001.

[4] Anastassiou, G.A., Intelligent Mathematics: Computational Analysis, Springer, Heidelberg, New York, 2011.

[5] Anastassiou, G.A., Nonlinearity: Ordinary and Fractional Approximations by Sublinear and Max-Product Operators, Springer, Heidelberg, New York, 2018.

[6] Bede, B., Coroianu, L., Gal, S., Approximation by Max-Product type Operators, Springer, Heidelberg, New York, 2016.

[7] Choquet, G., Theory of capacities, Ann. Inst. Fourier (Grenoble), 5(1954), 131-295.

[8] Denneberg, D., Non-additive Measure and Integral, Kluwer, Dordrecht, 1994.

[9] Korovkin, P.P., Linear Operators and Approximation Theory, Hindustan Publ. Corp. Delhi, India, 1960.

[10] Podlubny, I., Fractional Differentiation Equations, Academic Press, San Diego, New York, 1999.

[11] Schmeidler, D., Integral representation without additivity, Proceedings of the American Mathematical Society, 97(1986), 255-261.

[12] Shisha, O., Mond, B., The degree of convergence of sequences of linear positive operators, Nat. Acad. of Sci. U.S., 60(1968), 1196-1200.

[13] Wang, Z., Klir, G.J., Generalized Measure Theory, Springer, New York, 2009.

George A. Anastassiou

Department of Mathematical Sciences

University of Memphis

Memphis, TN 38152, U.S.A.

e-mail: ganastss@memphis .edu 